\title{
Clinical Trial Subject Identification Log
}

National Cancer Institute

\section{Source}

National Cancer Institute. Clinical Trial Subject Identification Log. NCI Thesaurus. Code C125434.

A report or log that fully identifies all clinical trial subjects screened in a clinical trial. 